\title{
Acknowledgement of reviewers for 2021
}

\author{
Mariano García-Fernández
}

Published online: 5 February 2022

(C) The Author(s), under exclusive licence to Springer Nature B.V. 2022

Please join us in thanking all those scientists and experts in the various fields represented in Journal of Seismology for devoting time and effort to review the papers that we have been sending them. The Editor-in-Chief and Publisher acknowledge the colleagues listed below for their excellent reviews of papers for which final decisions have been made during the period 1 January 2021 to 31 December 2021.

Robert Abbott
Shubham Agrawal
Ozgenc Akin
Dario Albarello
Richard Alfaro-Diaz
Charles Ammon
Abdolrasool Anooshehpoor
Iman Ashayeri
Josep Batllo
Christian Beck
Dirk Becker
Dino Bindi
Antonella Bobbio
Petros Bogiatzis
M. Bohnhoff
Julian Bommer
István Bondár
Luis Fabian Bonilla
Fabio Bruni
Fabio Bruni
Eric Calais
Frédéric Cappa

Alexandra Carvalho
Marco Cattaneo
Carlo Cauzzi
Calum Chamberlain
Tianjian Chen
Feng Cheng
Sumer Chopra
Konstantinos Chousianitis
Maxime Claprood
David Cornwell
Stephen Crane
Neala Creasy
Saumik Dana
Eric Daub
Pasquale De Gori
Raffaella De Matteis
Angelo De Santis
Vincenzo del Gaudio
Jose Delgado
Boumediene Derras
Domenico Di Giacomo
Nuno Dias

Jordi Diaz

Steve Dickenson

Laure Duboeuf

Ahmed Elbanna

Kentaro Emoto

Läslo Evers

Hortencia Flores Estrella

Umberto Fracassi

Wolfgang Friederich

Simona Gabrielli

Vineet Gahalaut

Kalpna Gahalaut

Odysseus Galanis

Pauline Galea

Juan Galiana-Merino

Jesús Galindo-Zaldivar

Mariarosaria Gallipoli

Danilo Galluzzo

Athanassios Ganas

Alexander Garcia

Antonio Garcia-Jerez

Paolo Gasperini 


Stefania Gentili
Elisabetta Giampiccolo
Richard Gibson
Alessandro Giocoli
Roberta Giuliani
Stefan Glazer
Rengin Gok
Álvaro González
Robert Graves
Gottfried Grünthal
Annabel Haendel
Sebastian Hainzl
Miroslav Hallo
Jens Heinicke
Ross Heyburn
Hector Hinojosa
Klaus-G. Hinzen
Ben Holtzman
Roser Hoste-Colomer
Jinjun Hu
Zhouchuan Huang
Antonio Giovanni Iaccarino
Ryoya Ikuta
Tahir Serkan Irmak
Anthony Jamelot
Steven C. Jaume
Chunyang Ji
Sun Jianbao
Maria-Jose Jimenez
Umar Kadiri
Ronnie Kamai
Charu Kamra
Honn Kao
Savas Karabulut
George Karakaisis
Vassilis Karakostas
Ioannis Kassaras
Hiroshi Kawase
Robert Kayen
Sarfraz Khan
Naeem Khoshnevis
Andrzej Kijko
Eduard Kissling
Peter Klin
Sreevalsa Kolathayar
Michael Korn
Masahiro Kosuga
Edward Krebes

Daniela Kühn

Dinesh Kumar

Mario La rocca

Horst Langer

Giovanni Lanzano

Marine Laporte

Stanislav Lasocki

Cédric Legendre

Denis Legrand

Ved Lekic

José V. Lemos

Xiaojun Li

Wei Liu

Grzegorz Lizurek

Andrea Llenos

John Louie

Richard Luckett

Quanbo Luo

Colton Lynner

Alessia Maggi

Sujith Mangalathu

Brassnavy Manzunzu

Shmuel Marco

Basil Margaris

Giovanni Martinelli

Claudia Mascandola

Eric Matzel

George Mavroeidis

Yves Mazabraud

John McBride

Mustapha Meghraoui

Francesco Mele

Nikolaos Melis

Andrew Michael

Arnaud Mignan

Giuliano Milana

Hiroe Miyake

Juan Montalvo

Melissa Moore

Aurelien Mordret

Seyed Mostafa Mousavi

Francesco Mulargia

Roger Musson

Sahar Nazeri

Sanjay Nimbalkar

Arda Ozacar

Francesca Pacor

Passakorn Pananont

Francesco Panzera
Eleftheria Papadimitriou

Gerassimos Papadopoulos

Imtiyaz Parvez

Arthur Pate

Anne Paul

Florin Pavel

Antonella Peresan

Floriana Pergalani

Gesa Maria Petersen

Matteo Picozzi

Vyacheslav Pilipenko

Valerio Poggi

Natalia Poiata

Hongrui Qiu

Neta Rabin

Mehdi Raoofian-Naeeni

Karthik Reddy

Lise Retailleau

Adam Ringler

Graciela Rojo Limon

Pamela Roselli

Zafeiria Roumelioti

Pierre-Francois Roux

Georg Rümpker

Fabio Sabetta

Amos Salamon

Meghdad Samaei

Francisco Sánchez-Sesma

Angela Saraò

Joachim Saul

Roberto Scarpa

Martin Schimmel

Gretchen Schmauder

Frederic Schoenberg

Oona Scotti

Vincenzo Serlenga

Blessing Shumba

Livio Sirovich

Andreas Skarlatoudis

Megan Slinkard

Efthimios Sokos

Alexandre Soloviev

Mathilde Sorensen

Tony Stabile

Don Steeples

Brian Stump

Matteo Taroni

Dennise Templeton

Timo Terasvirta 
Jeremy Thomas

Christel Tiberi

Frederik Tilmann

Fumiaki Tomita

Mehdi Tork Qashqai

Patrizia Tosi

Theofilos Toulkeridis

Michael Tserasky
Ergin Ulutas

Filippos Vallianatos

Koen Van Noten

Brigitte Van Vliet Lanoe

Marc Wathelet

Nadav Wetzler

Jan Wiszniowski

Shunroku Yamamoto
Hiroaki Yamanaka

Qin Yan

Hamid Zafarani

Chuanbin Zhu

Moritz Ziegler

Dimitri Zigone

We apologize for any errors or inadvertent omissions.

Mariano García-Fernández

MNCN-CSIC, Madrid, Spain

Email: mariano.garcia@csic.es
Publisher's note Springer Nature remains neutral with regard to jurisdictional claims in published maps and institutional affiliations. 